\title{
NEW TYPE OF PERIODIC BURSTS OF NON-IO JOVIAN DECAMETRIC RADIO EMISSION
}

\author{
M. Panchenko*, and H. O. Rucker*
}

\begin{abstract}
Analyzing the data acquired by STEREO/WAVES, Wind/WAVES and Cassini/ RPWS instruments during the time interval between the years 2002-2010 we have revealed unusual periodic radio bursts of the non-Io controlled component of the Jovian decametric radiation (non-Io DAM). These non-Io bursts are typically observed in a frequency range from $\sim 5 \mathrm{MHz}$ up to $\sim 10-16 \mathrm{MHz}$ and recur during several Jovian days with a surprisingly new period of $\sim 10.07$ hours. This period is $1.5 \%$ longer than the rotation period of the inner Jovian magnetosphere (System III, 9.925 hour). The occurrence probability of these periodic bursts has been found to be significantly higher in the sector of Jovian Central Meridian Longitude between $300^{\circ}$ and $60^{\circ}$ (via $360^{\circ}$ ), corresponding to the region of non-Io-C sources. Stereoscopic observations performed by STEREO/WAVES as well as Wind/WAVES and Cassini/RPWS suggest that the sources of the periodic bursts sub-corotate with Jupiter. The relations between the occurrence of the periodic bursts and solar wind activity have been analyzed.
\end{abstract}

\section{Introduction}

Jupiter with the largest planetary magnetosphere in the solar system is a complex source of a powerful coherent non-thermal radio emission [Zarka, 1998; Zarka and Kurth, 2005, and references therein]. This emission is attributed to the mechanism of the electron cyclotron maser instability [Wu and Lee, 1979] in which the radiation is generated by the accelerated electrons characterized by an instable distribution function, e.g. loss cone or shell distributions.

Decametric radio emission (DAM), the strongest component of Jovian radiation discovered more than 50 years ago by Burke and Franklin [1955], is observed in a form of arc shaped radio bursts (in a minute timescale) in a frequency range from few $\mathrm{MHz}$ up to $40 \mathrm{MHz}$ [Carr et al., 1983; Zarka, 1998]. The occurrence rate as well as total intensity of the Io independent component of the DAM (non-Io DAM) exhibits strong modulation periodicity

* Space Research Institute, Austrian Academy of Sciences, Schmiedlstrasse 6, A-8042 Graz, Austria 
related to the rotation of the Jupiter's magnetosphere (9.9249 h, System III) due to a non-axisymmetric magnetic field of Jupiter and non-isotropic emitting directivity of DAM [Carr et al., 1983; Kaiser, 1993]. The other components of Jovian radiation also exhibit several strong periodicities. Several studies have shown that the occurrence of a narrowband Jovian kilometric radiation $(\mathrm{nKOM})$ as well as the intensity of the hectometric Jovian radiation (HOM) are modulated with a period slower by $3 \%-5 \%$ than System III [Kaiser and Desch, 1980; Kaiser et al., 1996]. Kaiser et al.[1996] also suggested the existence of a high density region in the Io plasma torus. This region rotates with a System IV period [Sandel and Dessler, 1988] and can block the HOM emission at times when the observer is located at the same longitudes with this high density spot.

Generally, non-Io DAM is a highly variable and sporadic radio burst component. Nevertheless, analyzing the radio spectra recorded by the two STEREO/WAVES spacecraft [Bougeret et al., 2008], Wind/WAVES [Bougeret et al., 1995] and Cassini/RPWS [Gurnett et al., 2004] we have found intensive radio bursts, not previously reported, which recurred very periodically during several Jupiter's days with a surprisingly new period of $\sim 10.07$ hours [Panchenko et al., 2010]. In this paper we review the main observational properties of these periodic radio bursts. Additionally, we analyze the influence of the solar wind on the occurrence of the periodic bursts.

\section{Periodic non-Io DAM Radio Bursts and its Properties}

During the period of time between years 2002-2010 several spacecraft missions including those orbiting Earth and the Sun are able to receive the Jovian radio emission in frequency ranges from few $\mathrm{kHz}$ up to $14-16 \mathrm{MHz}$. We have analyzed dynamic radio spectra of Jovian decametric radio emission acquired by STEREO/WAVES, Wind/WAVES and Cassini/ RPWS. The special interest represents simultaneous multi-spacecraft stereoscopic observation of the DAM. In contrast to the observations from a single point, the stereoscopic measurements facilitate unambiguous recognition of the Jovian decametric radio emission in the observed dynamic spectra as well as identification of its components. Using the fact that Jovian radio emission is emitted in a hollow cone attached to the Jovian magnetic field or to the Io flux tube and the known rotation rate of Jovian magnetosphere $(9.925 \mathrm{~h})$ or Io's orbital period (42.46h), the DAM emission can be identified by means of the time delay between sequential detection of the radio emission from the same radio source by two spacecraft separated in space (see Panchenko et al.[2010]).

Analyzing the STEREO /WAVES, Wind /WAVES and Cassini /RPWS measurements of Jovian DAM we have found radio bursts which recurred very periodically during several Jupiter rotations (see examples in Figure 1). These bursts were typically detected within a frequency range from about $5 \mathrm{MHz}$ to about $12 \mathrm{MHz}$ or in some cases more than $16 \mathrm{MHz}$ - the higher frequency limit of the STEREO/WAVES instrument. The periodic radio bursts have an intensity comparable to the 'typical' Jovian DAM 'arc-like' radio emission and in some cases these features were very similar to vertex late arcs of nonIo DAM [Queinnec and Zarka, 1998]. The duration of each periodic burst at the same frequency was several minutes. By means of the stereoscopic observations (see Panchenko et al.[2010]) these periodic radio bursts have been identified as non-Io DAM. In particular 

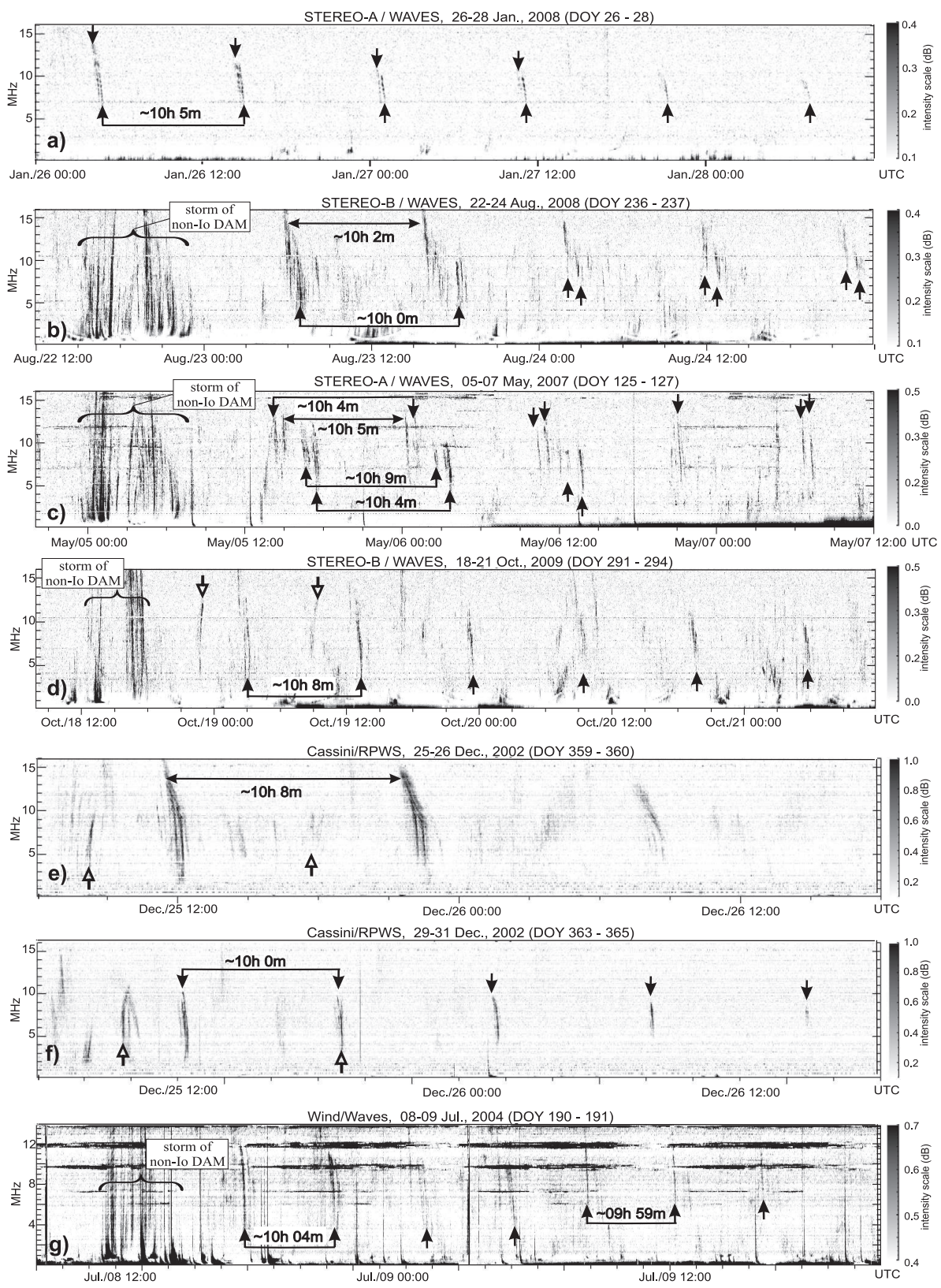

Figure 1: Examples of the periodic burst observed by STEREO/ WAVES, Cassini/ RPWS and Wind/ WAVES. Arrows mark different groups of the periodic burst during one episode. The average period of the burst repetition in each group is given. Curly brackets in panels $b, c, d$ and $g$ indicate the intensive storms of the non-Io DAM. 
the time delay (after correction on light travel time from Jupiter to each of the spacecraft) between sequential detection of the same bursts onboard STEREO-A and STEREO-B as well as Wind and Cassini corresponds to the time for Jupiter to rotate through the angular spacecraft separation.

Almost all detected periodic bursts have very similar spectral features: small negative frequency drift and "burst-like" structure. In general, all the observed periodic bursts can be divided into several morphological groups: single bursts (Fig. 1d, 1f, 1g), group of multiple bursts (Fig. 1a), several groups of single or multiple bursts (Fig. 1b, 1c) and more complex periodic structures consisting of the single and multiple bursts (Fig. 1e). We have also found a few episodes with positive frequency drift (Fig. 1d, 1e, and 1f, open arrows) as well as with periodicity exactly corresponding the Jovian System III period. Nevertheless, the number of such episodes is insufficient to draw the conclusion whether these periodic features really exist or not.

Stereoscopical observations allow also to define if the observed radio emission modulated by the planet rotation is originated from a "clock-like" (or stroboscopic) source, similar to Saturnian SKR, or this emission is modulated due to with the planet co-rotating radio emission beam (e.g. Jovian n-KOM emission). Thus, we found several episodes when the same periodic bursts were detected stereoscopically onboard Wind and Cassini spacecraft separated in space by the relative large angular distance, e.g. about $90^{\circ}$. In this case the periodic bursts were observed sequentially with proper time delay (more than 2 hours) which was close to the Jupiter time rotation through angular separation of the spacecraft. This strongly suggests that the sources of the periodic bursts sub-corotate with Jupiter and may be active during relative longer period of time (maximal periodic bursts were detected during 7 Jupiter rotations).

\subsection{Statistics of the Events}

In total, 66 episodes of periodic bursts (or 227 individual bursts) have been detected in dynamic radio spectra recorded by STEREO/WAVES, Wind/WAVES and Cassini/ RPWS during the years 2002-2010 (one episode means continuous repetition of the periodic structures in the dynamic spectra). In particular, the STEREO /WAVES observed 37 episodes (117 individual bursts) during 2007-2010, Wind/WAVES detected 22 episodes (88 bursts) during 2004-2006, and Cassini /RPWS recorded 7 episodes(22 bursts) in the years 2002-2003.

For each episode the time interval between individual bursts repetition within each group have been determined. Moreover, we have defined a spacecraft Jovian Central Meridian Longitude (CML) and Io-phase at the time when each particular burst was observed. Determining the period of the burst repetition we have also accounted for possible Doppler shifting of the period caused by the motion of spacecraft along its orbit relative to the rotating Jupiter. A very simple calculation shows that such effect may lead to an error in the period determination which is less than the time resolution of the used spaceborn radio instruments.

Figure 2 shows a histograms of the distributions of the determined periods of the bursts reoccurrence (time between pairs of consecutive bursts) as well as CML and Io phase of 

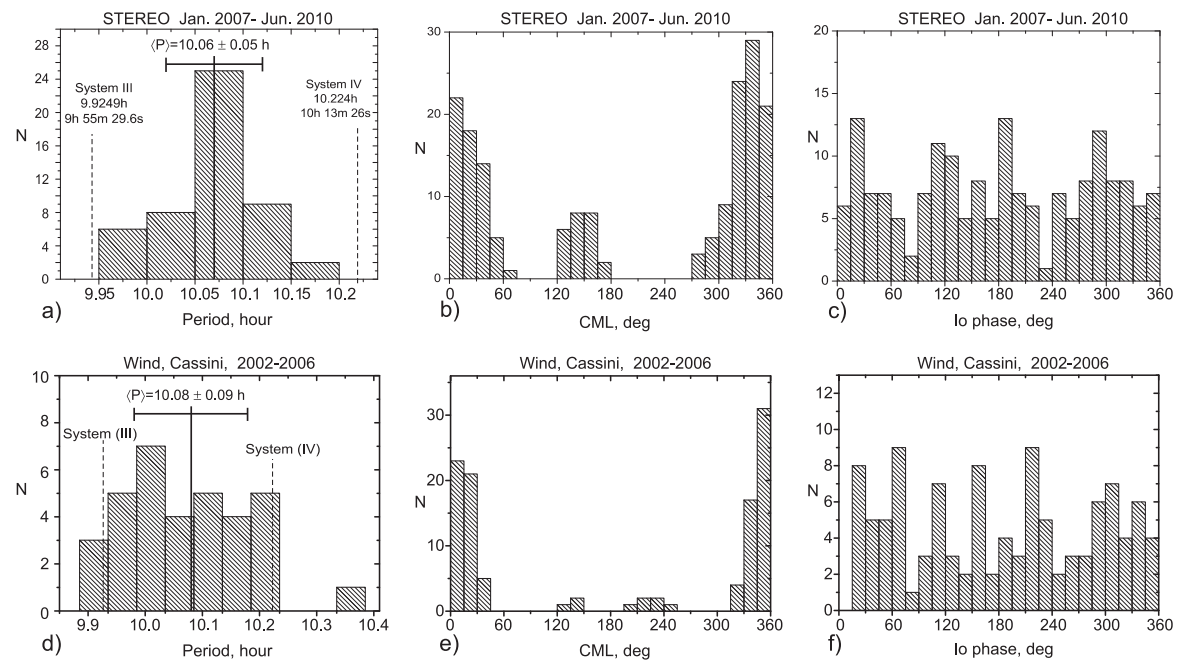

Figure 2: Statistical results of observations acquired by STEREO/WAVES (117 bursts in 37 episodes, top panels) and Wind/WAVES and Cassini/RPWS (110 bursts in 29 episodes, bottom panels). Panels a) and d) show histograms of the distribution of the determined periods of the burst repetition. The horizontal lines centered about $10.06 \mathrm{~h}$ and $10.08 \mathrm{~h}$ denote one sigma error of the mean values. Distribution of the CML (III) and Io phases of the spacecraft at times of detection of each burst are presented in panels b), e) and c), f) respectively.

the spacecraft in the moment of the detection of each particular burst. It is clearly seen that all bursts recurred with periods longer than System III but shorter than averaged period of System IV (10.224). In particular, the histogram in Fig. 2a which shows the distribution of the bursts periods measured by STEREO/WAVES has a very clear peak at 10.06h. In case of Wind/WAVES and Cassini/RPWS (Fig. 2d) no well pronounced peak is visible. Most probably, this is due to the relative lower sensitivity of the Wind/ WAVES instruments (22 out of 29 episodes in the statistics of Fig. 2d was observed by Wind/WAVES) which results in a lower accuracy of the determined periods. Nevertheless, similar to STEREO observations, most of the observed bursts reoccurred with periods in the range between Jovian System III and System IV rate. Out of the observations the averaged period of the bursts reoccurrence is $10.07 \pm 0.01$ hours $(10 \mathrm{~h} 4 \mathrm{~m} \pm 6 \mathrm{~m}$ ), which is $\sim 1.5 \%$ longer than System III.

Figures 2b, 2c, 2e and $2 \mathrm{f}$ show a histogram of CML (III) positions and Io phases of the spacecraft at times of detection of each radio burst. We note the strong correlation between the spacecraft CMLs and the occurrence of the periodic bursts (histograms $2 \mathrm{~b}$ and $2 \mathrm{e})$. Most of the periodic bursts $(\sim 90 \%)$ were detected when the spacecraft were between $300^{\circ}$ and $60^{\circ}$ (via $360^{\circ}$ ) of CML (III). This CML range corresponds to source locations of the non-Io-C DAM (see e.g. Carr et al. [1983]). Only a few episodes were detected at around $180^{\circ}$ of CML. These results suggest the existence of 'active longitudes' or preferable locations of the sources of the periodic bursts where the mechanism of 
generation is more efficient. This is similar to the existence of the regions along the Io's orbit with respect to the Jovian magnetic field (Io's active longitudes) where the Io-DAM occurs more often [Carr et al., 1983].

In the same time from the Io phase histogram (Figure $2 \mathrm{c}$ and $2 \mathrm{f}$ ), we can conclude the absence of any correlation between the position of Io and occurrence of the periodic bursts. This result supports the suggestion that the observed periodic bursts are an Io independent component of the DAM.

\subsection{Anisotropy of the Beam Pattern}

One of the interesting features of the periodic bursts is a strong anisotropy of its beam pattern. The emission pattern of the Jovian DAM is believed to have a form of a thinwalled hollow cone attached to an instant magnetic field line [Carr et al., 1983]. Assuming that periodic bursts have the same beaming pattern and having in mind the fact that the sources of these bursts nearly co-rotate with the inner magnetosphere we would expect to observe the radio burst twice per rotation period of Jupiter similar to vertex-early and vertex-late arcs of Io-DAM. Nevertheless, generally only one burst per Jupiter rotation (as seen in Fig. 1) was observed. Only in a few events significantly weaker second bursts with a small positive frequency drift (like vertex-early) were observed in a pair with the "main" burst (e.g. Fig. 1d, Fig. 1e and Fig. 1f, marked by open arrows). The absence of the second burst per rotation period may indicate a strong anisotropy of the emission cone pattern, when the intensity of the radiation depends on an angle with respect to a symmetry axis. Examples of such anisotropy have been found for the terrestrial Auroral Kilometric radiation (AKR). Mutel et al. [2008] have shown that individual bursts of AKR radiate not in cones but rather in a narrow plane tangent to the source's magnetic latitude. As an alternative model of the DAM beaming Imai et al.[2008] suggested that the Jovian DAM propagates in a narrow beam like a searchlight. Recently Galopeau and Boudjada [2010] have introduced a model in which the cone of the DAM emission is non-axi-symmetrical, i.e. flattened in the direction of the local magnetic field. Such anisotropy beaming patterns may suggest the strong not axisymmetrical amplification of the emission in the source or the existence of a specific plasma environment in the emission source regions. For example, the "tangent plane" model of AKR beaming proposed by Mutel et al. [2008] requires that the radio source should be located in the plasma density cavity, analogous to the AKR. Such cavities are not yet detected in the Jovian magnetosphere.

\subsection{Solar Wind Control}

The solar wind control on the non-Io component of DAM emission is known since the Voyager missions (e.g. Barrow and Desch [1989], Genova et al. [1987]). The direct measurements have also shown that part of the non Io-DAM and strong UV auroral emission are triggered by interplanetary shocks [Gurnett et al., 2002], although the mechanism of solar wind impact is still unknown. 


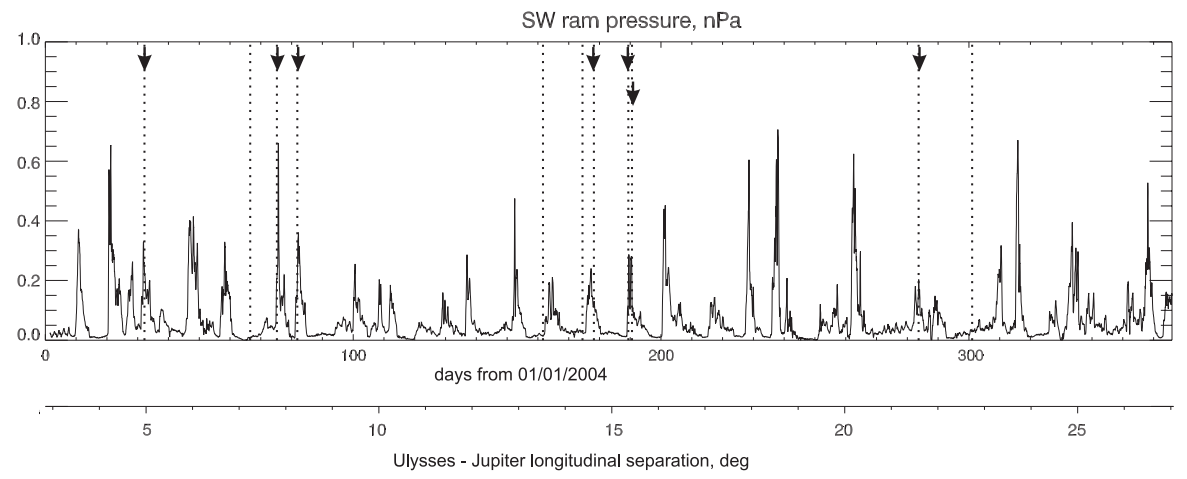

Figure 3: Solar wind ram pressure measured by Ulysses/SWOOPS and ballistically propagated to Jupiter orbit. The vertical dotted lines indicate the beginning of the episodes of periodic bursts detected by Wind/WAVES. The bottom axis shows the longitudinal separation between Ulysses and Jupiter.

The solar wind observation performed by Ulysses/SWOOPS [Bame et al., 1992] in the year 2004 and the corresponding Wind/WAVES radio data have been used to study the possible correlation between solar wind activity and the periodic bursts of the non-Io DAM. In 2004 the Ulysses spacecraft had its second encounter with Jupiter and measured the solar wind parameters close to the ecliptic plane. These parameters have been ballistically propagated to the position of Jupiter. Figure 3 shows a time profile of the ballistically propagated solar wind ram pressure. The vertical dotted lines indicate the beginning of the periodic bursts events recorded by the Wind/WAVES. It is well seen that 7 out of 11 episodes (marked by arrows in Figure 3) are well correlated with the significant enhancement of the solar wind ram pressure.

Additionally, most of the episodes with periodic radio bursts were detected after storms of the non-Io DAM - sequences of the strong non-Io DAM bursts (see Fig. 1). In order to study the correlation between the occurrence of periodic burst and intensification of the sporadic non-Io DAM we have selected the episodes in which the periodic bursts appeared during the following $\sim 10$ hours after the strong non-Io DAM storm. To exclude the possible uncertainties in the identification of the radio emission (solar radio, Io controlled or Io independent DAM) we have used only stereoscopic STEREO/WAVES observations which, as was mentioned in the section 2 and [Panchenko et al., 2010], allow unambiguous recognition of the Jovian non-Io DAM in the dynamic spectra. The results have shown that in 25 out of 37 (or 68\%) episodes observed by STEREO/WAVES in years 2007-2010 the periodic bursts were detected after series of intensive bursts of the non-Io DAM (e.g. Fig 1b, 1c, 1d). Therefore we can conclude that the appearance of the periodic bursts is well correlated with intensification of the sporadic non-Io DAM emission (storms of the non-Io DAM) which is known to be significantly affected by the solar wind [Barrow and Desch, 1989; Genova et al., 1987]. 


\section{Discussion}

Taking into account the number of the observed episodes a existence of the new type of the periodic non-Io DAM radio bursts appears to be very likely. Having a longer active period of time (several Jupiter rotations) the periodic bursts are expected to be a reasonable tool for studying the beaming properties of the DAM as well as for the diagnostics of the plasma conditions which influence the generation, escape and propagation of the emission.

In general the generation of non-thermal radio emission via a mechanism of cyclotron maser requires energetic electrons accelerated along magnetic field lines. In case of Iocontrolled DAM the source of the hot electrons is believed to be the Io ionosphere. Based on the solar wind influence on the non-Io DAM it is widely believed that non-Io DAM sources, which have never been resolved from direct measurements, are located at high auroral latitudes which are connected to the middle or outer Jovian magnetosphere [Zarka, 1998]. However, there are studies which suggest also the low-latitude $(L<6)$ sources. Arkhypov and Rucker [2009] have shown that the periodicity of the non-Io storms coincide with the fundamental eigenmode of the Io torus oscillation and therefore part of the nonIo emission can be directly related to the plasma torus. Therefore one possible source candidate for the energetic particles which may produce the periodic burst of non-Io DAM is the Io plasma torus which consists of highly structured plasma regions. Io torus plasma corotates with Jupiter in the centrifugal equatorial plane with averaged $\approx 10.224$ hour period (System IV). In the same time Steffl et al.[2006] have reported that the azimuthal composition in the Io plasma torus, observed by Cassini/UVIS, exhibits the $\approx 10.07 \mathrm{~h}$ ( $1.5 \%$ longer than System III) variation. This rate is equal to the $10.07 \pm 0.09 \mathrm{~h}$ averaged period of the periodic bursts repetitions observed in the years 2002-2010. The weak point of the hypothesis that sources of the periodic bursts are related to the Io plasma torus, is the strong influence of the solar wind on the periodic burst occurrence and lack of such an impact on the Io dependent radio emission (e.g. Genova et al. [1987]). Another possible candidate to be a source of the energized particles which produce periodic bursts is the "middle" magnetosphere linked with the co-rotating "main aurora oval". This oval is thought to be connected with the magnetosphere-ionosphere coupling current system associated with the breakdown of rigid co-rotation in the middle magnetosphere [Cowley et al., 2003].

\section{Summary}

The main finding of the study reported here is the detection of a new periodicity in Jovian decametric radio emission. The results of the observation are as follows:

1. Periodic bursts have been detected in decametric wavelengths between 5 and 12-16 $\mathrm{MHz}$. The averaged period of the burst recurrence is $\sim 1.5 \%$ longer than System III $(9.925 \mathrm{~h})$.

2. The periodic bursts are a non-Io component of DAM and its sources sub-corotate with Jupiter and it may be active during longer periods of time. 
3. The probability of observing the periodic bursts was found to be significantly greater between $300^{\circ}$ and $60^{\circ}$ (via $360^{\circ}$ ) of $\mathrm{CML}$ (III). No correlation with the Io position as well as other satellites has been found.

4. The beam pattern of the periodic bursts exhibits strong anisotropy.

5. Occurrence of periodic bursts of non-Io DAM is correlated with solar wind conditions around Jupiter, i.e. the bursts started after enhancement of the solar wind ram pressure.

Acknowledgments. The authors are pleased to acknowledge the STEREO/WAVES and Plasma Physics Data Center (CDPP), Wind/WAVES, Cassini/RPWS and Ulysses/ SWOOPS teams for access to data. This work was financed by the "Austrian Fonds zur Förderung der wissenschaftlichen Forschung" (project P20680-N16).

\section{References}

Arkhypov, O.V., and H. O. Rucker, Sub-hour modulation of non-Io Jovian decametric emission, Astron. Astrophys., 497, 551-555, 2009.

Bame, S. J., D. J. McComas, B. L. Barraclough, J. L. Phillips, K. J. Sofaly, J. C. Chavez, B.E. Goldstein, and R.K. Sakurai, The ULYSSES solar wind plasma experiment, Astron. Astrophys. Suppl. Ser., 92, 237-265, 1992.

Barrow, C.H., and M.D. Desch, Solar wind control of Jupiter's hectometric radio emission, Astron. Astrophys., 213, 495-501, 1989.

Bougeret, J.-L., M. L. Kaiser, P. J. Kellogg, R. Manning, K. Goetz, S. J. Monson, N. Monge, L. Friel, C. A. Meetre, C. Perche, L. Sitruk, and S. Hoang, WAVES: The radio and plasma wave investigation on the WIND spacecraft, Space Sci. Rev., 71, 231, 1995.

Bougeret, J.-L., et al. (42 co-authors), S/WAVES: The Radio and Plasma Wave Investigation on the STEREO mission, Space Sci. Rev., 136, 487-528, 2008.

Burke, B. F., and K. L. Franklin, Observations of a variable radio source associated with planet Jupiter, J. Geophys. Res., 60, 213-217, 1955.

Carr, T.D., M.D. Desch, and J.K. Alexander, Phenomenology of magnetospheric radio emissions, in Physics of the Jovian Magnetosphere, edited by A. J. Dessler, 226-284, Cambridge University Press, Cambridge, U. K., 1983.

Cowley, S. W. H., E. J. Bunce, and J.D. Nichols, Origins of Jupiter's main oval auroral emissions, J. Geophys. Res., 108, 8002, 2003.

Galopeau, P., and M. Boudjada, Beaming cone of Io-controlled jovian decameter radio emission and existence of localized active longitude, in Planetary Radio Emissions VII, edited by H. O. Rucker, W.S. Kurth, P. Louarn, and G. Fischer, Austrian Academy of Sciences Press, Vienna, this issue, 197-204, 2011. 
Genova, F., P. Zarka, and C. H. Barrow, Voyager and Nançay observations of the Jovian radio-emission at different frequencies: Solar wind effect and source extent, Astron. Astrophys., 182, 159-162, 1987.

Gurnett, D. A., et al. (16 co-authors), Control of Jupiter's radio emission and aurorae by the Solar wind, Nature, 415, 985-987, 2002.

Gurnett, D. A., et al. (29 co-authors), The Cassini Radio and Plasma Wave investigation, Space Sci. Rev., 114, 1, 395-463, 2004.

Imai, K., L. Garcia, F. Reyes, M. Imai, J. R. Thieman, and M. Ikuta, A Searchlight Beam Model of Jupiter's Decametric Radio Emissions, AGU Fall Meeting Abstracts, B1673, 2008.

Kaiser, M. L., Time-variable magnetospheric radio emissions from Jupiter, J. Geophys. Res., 98, 18757-18765, 1993.

Kaiser, M. L., and M. D. Desch, Narrow band Jovian kilometric radiation: A new radio component, Geophys. Res. Lett., 7, 389-392, 1980.

Kaiser, M. L., M.D. Desch, and M.E. Brown, Evidence for an Io plasma torus influence on high-latitude Jovian radio emissions, J. Geophys. Res., 101, 13, 1996.

Mutel, R. L., I. W. Christopher, and J.S. Pickett, Cluster multispacecraft determination of AKR angular beaming, Geophys. Res. Lett., 35, L07104, 2008.

Panchenko, M., H. O. Rucker, M. L. Kaiser, O. C. St. Cyr, J.-L. Bougeret, K. Goetz, and S. D. Bale, New periodicity in Jovian decametric radio emission, Geophys. Res. Lett., 37, L05106, 2010.

Queinnec, J., and P. Zarka, Io-controlled decameter arcs and Io-Jupiter interaction, J. Geophys. Res., 103, 26649, 1998.

Sandel, B. R., and A. J. Dessler, Dual periodicity of the Jovian magnetosphere, J. Geophys. Res., 93, 5487, 1988.

Steffl, A. J., P. A. Delamere, and F. Bagenal, Cassini UVIS observations of the Io plasma torus. III. Observations of temporal and azimuthal variability, Icarus, 180, 124-140, 2006.

Wu, C.S., and L. C. Lee, A theory of terrestrial kilometric radiation, Astrophys. J., 230, 621-626, 1979.

Zarka, P., Auroral Radio Emissions at the Outer Planets: Observations and Theories, J. Geophys. Res., 103, 20159-20194, 1998.

Zarka, P., and W.S. Kurth, Radio wave emission from the outer planets before Cassini, Space Sci. Rev., 116, 371-397, 2005. 